\title{
Palmprint Recognition Using Data Field and PCNN ${ }^{*}$
}

\author{
Yanxia Wang, Jianmin Zhao, and Guanghua Sun \\ College of Mathematics, Physics and Information Engineering \\ Zhejiang Normal University \\ 688 Yingbin Road, Jinhua, Zhejiang Province, 321004 China \\ \{wangyx, zjm, sungh\}@zjnu.cn
}

\begin{abstract}
In this paper, an approach is proposed for palmprint recognition, which uses PCNN and data field theory to extract local statistical structure features of a palmprint. In the method, the data field theory is firstly introduced to obtain a relative palmprint data field, which enhances the palm line information. Then the relative data field is input into a PCNN. Next, the local statistical structure features with four values are extracted from each sub-region. At last, all of local statistic-structural feature vectors are weighted and combined into a long feature vector to represent the palmprint. Experiments show that the novel features can effectively characterize different palmprints.
\end{abstract}

Keywords: Palmprint recognition, data field, PCNN, feature extraction.

\section{Introduction}

Palmprint recognition uses the features of palmprints to identify a person, that is, principal lines, wrinkles, ridges, minutiae points, singular points and texture etc. [1].In recent years, researchers have proposed many palmprint feature extraction and matching methods, which can be grouped into four categories: structure-based, statistical-based, subspace-based and coding-based [2].

The structure-based methods mainly use the information of the direction and location of principal lines and wrinkles to represent the palmprint [3-4]. These methods are simple and intuitional, but the performance of recognition depends greatly on the edge detectors or broad line detector and several thin and weak palm lines, such as wrinkles, might be too vague for detection by a line detector. The second class approaches include the local statistical-based and global statistical-based methods [5-7]. These methods utilized the transform domain features which can effectively reduce the within class variances to improve the performance. But, in these approached, the size of a block and the strategy of dividing blocks have great

\footnotetext{
* This work is supported partly by Opening Fund of Top Key Discipline of Computer Software and Theory in Zhejiang Provincial Colleges at Zhejiang Normal University (No. ZSDZZZZXK27), the Zhejiang Province Natural Science Foundations of China (No. LQ13F020026) and the National Natural Science Foundations of China (No. 61272468 and No. 61272130).
} 
impact on the recognition results. The third class is subspace-based approaches [8-10]. The subspace coefficients are used to construct feature vectors. Subspacebased methods have successfully applied in palmprint recognition, but subspace is greatly dependent on the training set. Once the training set is subject to change, the whole training procedure will be repeated. The last class methods encode the response of a bank of filters into bitwise code [11-12]. The coding-based method usually has a lower memory requirement and a fast matching speed, and thus has been very successful in palmprint representation and matching, but the methods are sensitive to the translation and rotation of palmprint images.

In order to resolve these problems, we propose a palmprint recognition method of using image data field and PCNN to extract local statistical structure features.

The rest of the paper is organized as follows: A brief description of data field theory is given in Section 2. Section 3 gives a brief overview of PCNN. The proposed method in detail is described in Section 4. Section 5 reports the experimental results and analysis. Finally, the conclusion is given in Section 6.

\section{Data Field Theory}

In physics, a field is a mode of description of the interaction of material particles. With the concept of the field theory of physics, Li [13] proposed a new field, image data field. In the method, the pixels of an image are seen as the particles with certain mass in the 2D space and the interaction of these pixels makes up an image data field. Let $\mathrm{q}$ is a given pixel particle in the image space $\mathrm{L}, \forall \mathrm{q} \in L$, the potential value of pixel particle q produced on pixel particle $\mathrm{p}$ is defined as $\varphi^{q}(p)=m^{q} \bullet \exp \left(-\left(\text { dist }_{p q} / \sigma\right)^{2}\right)$, dist $_{p q}=\max \left(\left|x_{p}-x_{q}\right|,\left|y_{p}-y_{q}\right|\right)$.Here, $\sigma>0$ controls the interaction range of the pixel particle, that is the influence factor; $m^{q}$ is the intensity of field source; dist $_{p q}$ is the distance between the pixel $\mathrm{q}$ and $\mathrm{p} ; x_{p}, x_{q}, y_{p}, y_{q}$ are the horizontal and vertical ordinates of the pixel $\mathrm{p}$ and $\mathrm{q}$ respectively.

For the data field formed by all of pixel particles in the $\mathrm{L}$, the potential value of any a pixel $\mathrm{p}$ can be defined as $\varphi(p)=\sum_{\text {dist }_{p q} \leq \varepsilon} \varphi^{q}(p)$.

According to different masses, the absolute image data field and the relative image data field are obtained by $\mathrm{Wu}$ [14].If the mass is the difference of grayscales between the center pixel and the pixel influenced by it, then the relative image data field can be obtained:

$$
\mathrm{m}_{r}^{q}=|f(p)-f(q)|, \quad \varphi_{r}=\sum_{\text {dist }_{p q} \leq \varepsilon} m_{r}^{q} \bullet \exp \left(-\left(\text { dist }_{p q} / \sigma\right)^{2}\right)
$$

Here, $\mathrm{m}_{r}^{q}$ is the relative mass, respectively; $f(\bullet)$ is the function of the image grayscale. Fig. 1 shows an example of the data field. From the Fig.1, it can be found that the edge information is highlighted by the relative data field. The edge information of 
palmlines is what we need, so the relative image data field should be selected to use in our method.

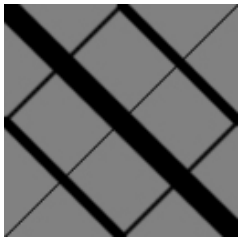

(a)

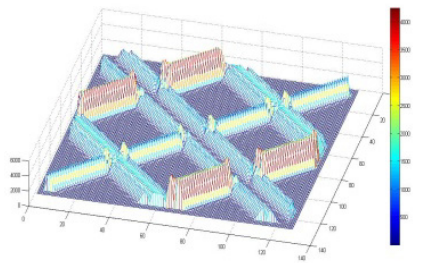

(b)

Fig. 1. Examples of image data fields. (a) Original image, (b) Relative image data field

\section{$3 \quad$ Pulse-Coupled Neural Network}

Pulse-coupled neural network is a called the 3 th generation neural network, which is a result of research on artificial neuron model that simulates the behavior of cortical neurons observed in the cortices of animal [15]. The significant advantage of the PCNN model is that it can operate without any pre-train needed. Other than that, it inherits the advantage of artificial neural network (ANN).

The PCNN neuron structure consists of three parts: the receptive field, the modulation field and the pulse generator. The dynamical behavior of neuron can be described as iteration by the following formulas:

$$
\begin{gathered}
F_{i j}[n]=S_{i j}+F_{i j}[n-1] e^{-\alpha_{F}}+V_{F} \sum_{k l} M_{i j k l} Y_{k l}[n-1] \\
L_{i j}[n]=L_{i j}[n-1] e^{-\alpha_{L}}+V_{L} \sum_{k l} W_{i j k l} Y_{k l}[n-1] \\
U_{i j}[n]=F_{i j}[n]\left(1+\beta L_{i j}[n]\right), \theta_{i j}[n]=\theta_{i j}[n-1] e^{-\alpha_{\theta}}+V_{\theta} Y_{i j}[n-1] \\
Y_{i j}[n]= \begin{cases}1, & U_{i j}[n]>\theta_{i j}[n] \\
0, & \text { Otherwise }\end{cases}
\end{gathered}
$$

Where $\mathrm{n}$ is current iteration number. The subscript $(i, j)$ is the position of a neuron in the network and a pixel in the input image. F, L, $\theta, \mathrm{U}$ and are feeding inputs, linking inputs, dynamic threshold, internal activity and pulse output, respectively. The status of the $(i, j)$ neuron can be indicated by $Y_{i j}$ with binary value. If $Y_{i j}$ equals to 1 , then it means the neuron is active. $\alpha_{F}, \alpha_{L}$ and $\alpha_{\theta}$ are decay time constants that control how the value of F,L and $\theta$ decay over time in the absence of external stimuli. $V_{F}, V_{L}$ and $V_{\theta}$ are normalizing constants that scale the inputs from adjacent pixels to their respective fields. $\mathrm{M}$ and $\mathrm{W}$ are the constant synaptic weight matrices and depended on the 
field of the neighborhood neurons. For our implementation, $\mathrm{M}=\mathrm{W} . \quad \beta$ is the strength of the linking. $\mathrm{S}$ is external input.

\section{The Main Idea}

In the method, the palmprint image is first mapped from gray space to data field space and a palmprint relative data field is obtained. PCNN is then applied to the entire relative data field. Subsequently, the local statistical structure features with four values are extracted from each sub-region with same size. At last, the all of local statistic-structural feature vectors are combined into a long feature vector to represent the palmprint. The processing flow is outlined in Fig. 2.

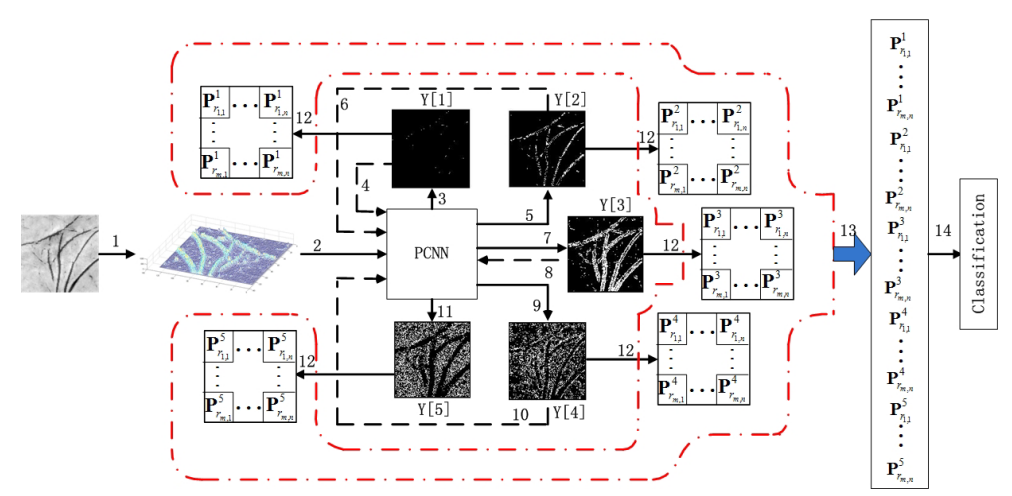

Fig. 2. Processing flow of the proposed method

In order to more effective represent the features of a palmprint, the binary output of each iteration is divided into small sub-regions with $16 \times 16$ size. Some statistics and the Tsallis entropy [16] of LBPH are computed from each sub-region and then they are composed of a local statistical structure feature vector, which can be described as:

$$
\begin{gathered}
\mathbf{P}=\left(\mathbf{P}_{r_{1,1}}^{1}, \mathbf{P}_{r_{1,2}}^{1}, \cdots, \mathbf{P}_{r_{m, n}}^{1}, \mathbf{P}_{r_{1,1}}^{2}, \mathbf{P}_{r_{1,2}}^{2}, \cdots, \mathbf{P}_{r_{m, n}}^{2}, \cdots, \mathbf{P}_{r_{1,1}}^{5}, \mathbf{P}_{r_{1,2}}^{5}, \cdots, \mathbf{P}_{r_{m, n}}^{5}\right) \\
\mathbf{P}_{r_{j, k}}^{i}=\left\langle n_{r_{j, k}}^{i}, \mu_{x r_{j, k}}^{i}, \mu_{y r_{j, k}}^{i}, T_{r_{j, k}}^{i}\right\rangle, \quad i=1, \cdots, 5, \quad j=1, \cdots, m, \quad k=1, \cdots n \\
m=\text { Height }_{F} / \text { Height }_{r}, \quad n=\text { Width }_{F} / \text { Width }_{r} \\
n_{r_{j, k}}^{i}=\sum_{x, y} Y_{r_{j, k}}^{i}(x, y), \quad Y_{r_{j, k}}^{i}(x, y)=\{0,1\} \\
\mu_{r_{j, k}}^{i}=\sum_{z \in r_{j, k}} x_{z} / n_{r_{j, k}}^{i}, \mu_{y r_{j, k}}^{i}=\sum_{z \in r_{j, k}} y_{z} / n_{r_{j, k}}^{i}, \quad Y_{r_{j, k}}^{i}(z)=1, \quad z(x, y) \in r_{j, k} \\
T_{r_{j, k}}^{i}=1-\sum_{d=0}^{255}\left(L B P H_{r_{j, k}}^{i}(d) / 255\right)^{q} /(q-1), \quad q>0
\end{gathered}
$$




$$
\begin{gathered}
L B P H_{r_{j, k}}^{i}(d)=\sum_{x, y \in r_{j, k}} I\left\{L B P_{r_{j, k}}^{i}(x, y)=d\right\}, \quad d=0,1, \cdots 255 \\
L B P_{r_{j, k}}^{i}(x, y)=\sum_{g=0}^{7} Y_{g}^{i}(x, y) 2^{g}, \quad Y_{r_{j, k}}^{i}(x, y) \in r_{j, k}^{i}, \quad Y_{g}^{i}(x, y)=\{0,1\}
\end{gathered}
$$

Where $Y_{r_{j, k}}^{i}(x, y)$ is the output of PCNN of the neuron $(x, y)$ in the $r_{j, k}$-th sub-region of the i-th iteration. Height ${ }_{F}$, Width $_{F}$, Height $_{r}$ and Width $r$ are the height and width of relative palmprint field and their sub-regions, respectively. $n_{r_{j, k}}^{i}$ is the number of the neuron of being fired in the $r_{j, k}$-th sub-region of the $i$-th iteration. $\mu_{x r_{j, k}}^{i}$ and $\mu_{y r_{j, k}}^{i}$ are the mean values of horizontal and vertical coordinates of all of fired neurons, respectively. $T_{r_{j, k}}^{i}$ is the Tsallis entropy of $L B P H_{r_{j, k}}^{i}$. Here, $L B P H_{r_{j, k}}^{i}$ is the local binary pattern histogram extracted from the $r_{j, k}$-th sub-region of the $i$-th iteration. $L B P_{r_{j, k}}^{i}(x, y)$ is the LBP operation of $Y_{r_{j, k}}^{i}(x, y)$.

\section{Experiment Results}

\section{$5.1 \quad$ Experiment Data}

In this paper, we develop a scanner-based device to capture palm images. The device is used to capture images of both hands from 300 individuals. Ten images are captured from each hand of a person. The center part of each palm is extracted and stored as palmprint images of size $128 \times 128$. A total of 6000 images $(300 \times 2 \times 10)$ form a palmprint database.

\subsection{Comparisons with other Methods}

In the experiment, some methods without any pre-train and our proposed method are tested on our palmprint database. The result of the proposed method is compared with the results of Local Gabor Binary Pattern Histogram Sequence (LGBPHS) method [18], DT-CWT based LBPWH method [10] and PCNN based method without using data field and these results are reported on Table 1.

Compared with the results of different method, it can be found that the recognition rate of the method of only using PCNN is $95.751 \%$, which is lower than that of other three methods. It because when the enhanced palmprint image is directly input into

Table 1. Recognition rates of different methods

\begin{tabular}{ccccc}
\hline Methods & LGBPHS & DT-CWT based LBPWH & only PCNN & Our method \\
\hline Results & $97.25 \%$ & $98.35 \%$ & $95.75 \%$ & $98.75 \%$ \\
\hline
\end{tabular}


PCNN, there is a white connected area in the output of PCNN. The area produced by the shadow of the palm center may make some useful information be covered and diminish the representation capabilities of their feature vectors. However, palm line information is directly enhanced by introducing relative data field in the proposed method. Therefore, our method gets the better performance than other three methods.

\section{$5.3 \quad$ Speed Test}

Speed is another factor in determining the efficiency of an algorithm. The proposed approach and other three methods are implemented using matlab 7.8 on a notebook personal computer with Intel core 2 processor $(2.13 \mathrm{GHz})$. Table 2 lists the average execution time of different methods.

Table 2. Execution time of different methods

\begin{tabular}{ccccc}
\hline Methods & LGBPHS & DT-CWT based LBPWH & only PCNN & Our method \\
\hline Results & $0.93 \mathrm{~s}$ & $0.88 \mathrm{~s}$ & $0.81 \mathrm{~s}$ & $0.84 \mathrm{~s}$ \\
\hline
\end{tabular}

From the Table 2, we can find that the execution time of all methods is very near. In our method, a relative palmprint data field needs to be constructed as the input of the PCNN. Therefore, our method needs more execution time. Even so, the execution time of our method is 0.84 second, which is fast enough for real-time verification. Thus, considering the various factors, our method applied in palmprint recognition gives the satisfying performance.

\section{Conclusion}

The paper reports a PCNN based local spatial structure feature extraction method for palmprint recognition. In order to enhance the information of palm lines, the method first introduces data field theory to construct the relative palmprint data field. Moreover, the local statistical structure features are extracted from not the single final output of PCNN but multiple FTMs in different iterations, which make the effective information increase. At last, all of local statistical structure features are composed to a final feature vector. The method is entirely independent on the training set. The proposed method is used to perform accuracy test and speed test. The experimental results demonstrate that the proposed approach can give a better performance. In summary, the PCNN based local statistical structure method is an effective feature extraction approach for palmprint recognition.

\section{References}

1. Zhang, D., Wai-Kin, K., You, J., Wong, M.: Online Palmprint Identification. Pattern Analysis and Machine Intelligence 25(9), 1041-1050 (2003)

2. Yue, F., Zuo, W.M., Zhang, D.: Survey of palmprint recognition algorithms. Acta Automatica Sinica 36(3), 353-365 (2010) 
3. Leung, F.L.M., Yu, X.: Palmprint matching using line features. In: Proceedings of the 8th International Conference on Advanced Communication Technology, Gangwon-Do, Korea, pp. 1577-1582 (2006)

4. Duta, N., Jain, A.K., Mardia, K.V.: Matching of palmprints. Pattern Recognition Letters 23(4), 477-485 (2007)

5. Kong, W., Zhang, D., Li, W.: Palmprint feature extraction using 2-D Gabor filters. Pattern Recognition 36, 2339-2347 (2003)

6. Jing, X., Zhang, D.: A face and palmprint recognition approach based on discriminant DCT feature extraction. IEEE Transactions on Systems, Man, and Cybernetics, Part B 34(6), 2405-2415 (2004)

7. Wang, Y., Ruan, Q.: Dual-tree Complex Wavelet Transform based Local Binary Pattern Weighted Histogram Method for Palmprint Recognition? Computing and Informatics 28, 299-318 (2009)

8. Lu, G., Zhang, D., Wang, K.: Palmprint recognition using eigenpalms features. Pattern Recognition Letters 24, 1463-1467 (2003)

9. Yang, J., Zhang, D., Yang, J., Niu, B.: Globally maximizing, locally minimizing: Unsupervised discriminant projection with applications to face and palm biometrics. IEEE Trans. Pattern Anal. Mach. Intell. 29(4), 650-664 (2007)

10. Wang, Y., Ruan, Q.: Kernel Fisher Discriminant Analysis for Palmprint Recognition. In: The Proceedings of the 18th International Conference on Pattern Recognion (ICPR 2006), vol. 4, pp. 457-460 (2006)

11. Kong, A., Zhang, D., Kamel, M.: Palmprint identification using feature-level fusion. Pattern Recog. 39(3), 478-487 (2006)

12. Zhang, D., Zuo, W., Yue, F.: A comparative study of palmprint recognition algorithms. ACM Computing Surveys 44(1), Article 2, 1-37 (2007)

13. Li, D.: Uncertainty in Knowledge Representation. Engineering Science 2(10), 73-79 (2000) (in Chinese)

14. Wu, T., Qin, K.: Image segmentation using cloud model and data field. Pattern Recognition and Artificial Intelligence 25(3), 397-405 (2012)

15. Eckhorn, R., Reitboeck, H.J., et al.: Feature linking via synchronous among distributed assemblies: simulations of results from cat visual cortex. Neural. Comput. 2, 1253-1255 (1990)

16. Zhang, Y., Wu, L.: Pattern recognition via PCNN and Tsallis entropy. Sensors 8 , 7518-7529 (2008)

17. Ojala, T., Pietikainen, M., Harwood, D.: A comparative study of texture measures with classification based on feature distributions. Pattern Recognition 29, 51-59 (1996)

18. Zhang, W., Shan, S., Gao, W., et al.: Local Gabor Binary Pattern histogram Sequence (LGBPHS): A Novel Non-Statistical Model for Face Representation and Recognition. In: Proceedings of the Tenth IEEE International Conference on Computer Vision (ICCV 2005), vol. 1, pp. 786-791 (2005) 